\title{
Assessment of Salivary Nitric Oxide Levels in Elite University Athletes in Japan: Findings From a Cross Sectional Study Design
}

\author{
Ryota Sone $^{\mathrm{a}}$, Kai Matsuba ${ }^{\mathrm{b}}$, Rei Tahara ${ }^{\mathrm{c}}$, Nobuhiko Eda ${ }^{\mathrm{d}}$, Keisei Kosaki ${ }^{\mathrm{e}}$, \\ Subrina Jesmin ${ }^{\mathrm{e}}$, Shumpei Miyakawa ${ }^{\mathrm{e}}$, Koichi Watanabe ${ }^{\mathrm{e}} \mathrm{f}$
}

\begin{abstract}
Background: High-intensity exercise affects the level of salivary nitric oxide (NO) with an impact on oxidative stress such as a reactive nitrogen-oxide species. However, in athletes with high-intensity training, the relationship between salivary NO levels and oxidative stress is yet to be clear. Additionally, the association of salivary NO levels and the common health disorders of athletes is unknown. Thus, the aim of this cross-sectional study was to clarify the relationship between salivary NO levels and oxidative stress, and the health/medical disorders existing in elite class university athletes.
\end{abstract}

Methods: In 250 athletes (males, 151 and females, 99) from undergraduate levels of Japanese University, we investigated the relationship between levels of salivary NO and oxidative stress markers: derived reactive oxygen species (d-ROMs) and biological antioxidant potential (BAP), and also examined that whether salivary NO levels are associated with diseases.

Results: There were no significant association between the levels of salivary NO and oxidative stress markers (such as d-ROM and BAP). From the questionnaire, asthma was the most prevalent as evident from medical history of the athletes. Additionally, the salivary NO levels were higher $(520 \pm 43 \mu \mathrm{mol} / \mathrm{L}$ vs. $375 \pm 13 \mu \mathrm{mol} / \mathrm{L}, \mathrm{P}<0.05)$ in the asthma group $(n=9)$ than in the non-asthma group $(n=241)$. We determined the optimal cut-off value $(\mathrm{P}=0.019)$ of the salivary NO levels for asthma was $425 \mu \mathrm{mol} / \mathrm{L}$, with a sensitivity of $88.9 \%$ and

Manuscript submitted October 22, 2018, accepted December 4, 2018

${ }^{\mathrm{a}}$ Graduate School of Comprehensive Human Sciences, University of Tsukuba, 1-1-1 Tennodai, Tsukuba, Ibaraki 305-8577, Japan

${ }^{\mathrm{b}}$ Tsukuba Sports Medicine and Health Science Center, University of Tsukuba Hospital, 2-1-1 Amakubo, Tsukuba, Ibaraki 305-8576, Japan

cSports Research and Development Core, University of Tsukuba, 1-1-1 Tennodai, Tsukuba, Ibaraki 305-8577, Japan

${ }^{\mathrm{d} F a c u l t y ~ o f ~ S p o r t s ~ S c i e n c e s, ~ W a s e d a ~ U n i v e r s i t y, ~ 2-579-15 ~ M i k a j i m a, ~ T o k o r o-~}$ zawa, Saitama 359-1192, Japan

'Faculty of Health and Sports Sciences, University of Tsukuba, 1-1-1 Tennodai, Tsukuba, Ibaraki 305-8577, Japan

${ }^{f}$ Corresponding Author: Koichi Watanabe, Faculty of Health and Sport Sciences, University of Tsukuba, 1-1-1 Tennodai, Tsukuba, Ibaraki 305-8577, Japan. Email: watanabe.koichi.ga@u.tsukuba.ac.jp

doi: https://doi.org/10.14740/jocmr3670 specificity of $61.8 \%$ (area under the curve (AUC), 0.73).

Conclusions: Our results suggest that the high levels of salivary NO in trained university athletes in Japan may potentially predict asthma. And this salivary NO level is not associated with markers of oxidative stress and existing diseases in athletes studied here.

Keywords: Nitric oxide; Oxidative stress; Asthma; Condition marker; Japanese athletes

\section{Introduction}

Nitric oxide (NO) is synthesized from the conversion of Larginine to L-citrulline by NO synthase (NOS) [1]. One of the three isoforms of NOS is inducible NOS (iNOS or NOS2), which is expressed in response to bacteria and proinflammatory stimuli and produces large amounts of NO for a longer period. NO, which is produced during host defense and immunologic reactions, is associated with nonspecific immunity [2]. Moreover, the nitrite and NO formed in the human oral cavity can be oxidized by salivary peroxidase $[3,4]$. Therefore, salivary NO affects oxidative stress as a reactive nitrogen oxide species (RNOS). In fact, several studies have reported high salivary NO levels in the patients with rheumatoid arthritis [5] and chronic periodontitis [6]. On the other hand, other studies suggested that the circulating levels derived from reactive oxygen metabolites (d-ROM) could serve as a biomarker of inflammation and disease severity in patients with rheumatoid arthritis [7]. In addition, biological antioxidant potential (BAP) was reported to be an oxidative stress marker related to antioxidant capacity [8]. However, cross-sectional research to clarify the relationship between levels of salivary $\mathrm{NO}$ and oxidative stress markers (d-ROM and BAP) and past history of illness in athletes has not been conducted yet.

Competitive athletes can easily fall into poor physical condition by daily high-intensity training, which affects their performance. The relationship between immune function and exercise has been demonstrated to decrease the risk of respiratory tract infection (RTI) by continuous moderate exercise training [9]. By contrast, continuous intensive training increases the risk of RTI in athletes [10]. In addition, even 
Table 1. Characteristics of the Study Subjects and the Sex-Related Differences

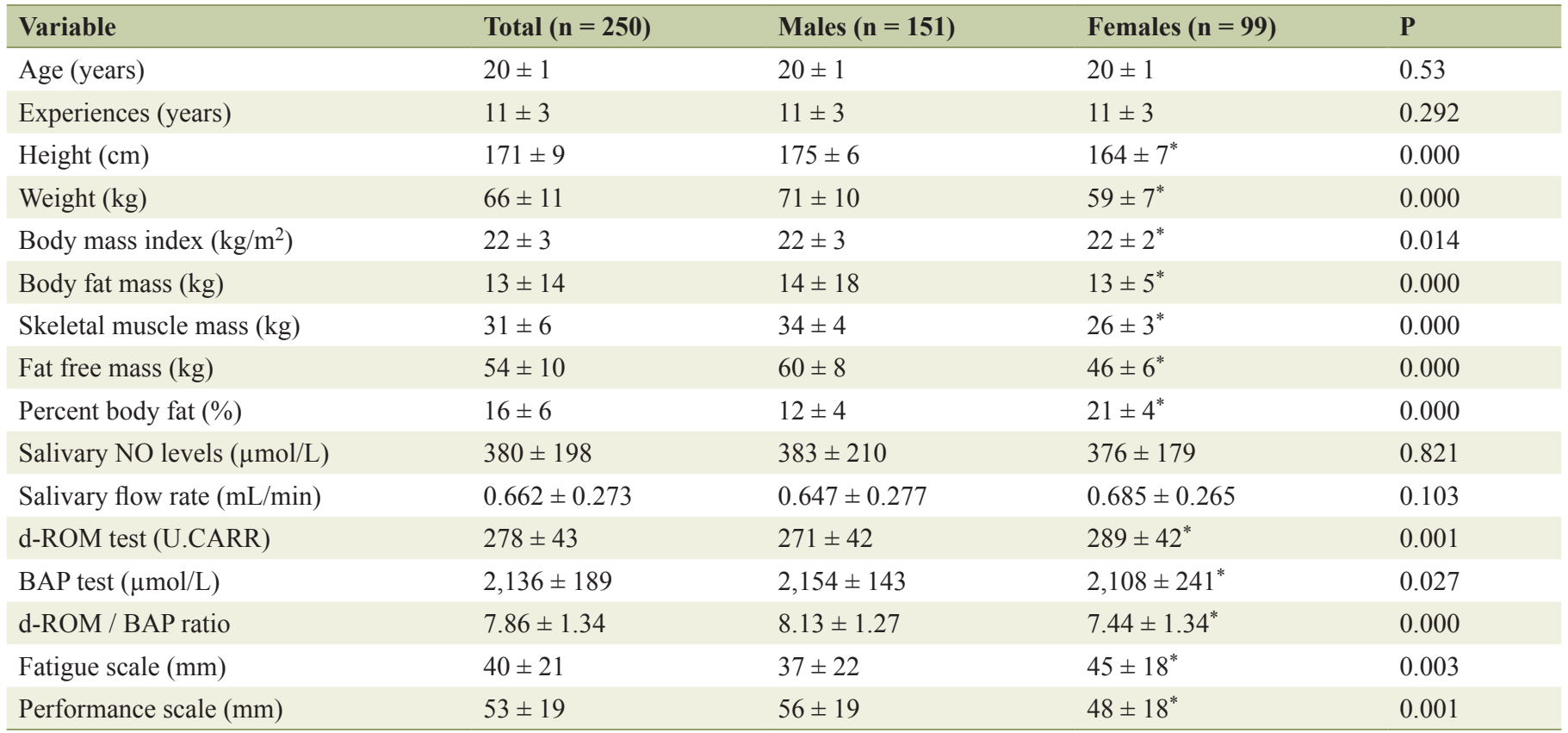

Data are shown as the mean \pm SD of the mean. ${ }^{*} P<0.05$, versus males. NO: nitric oxide; $d-R O M$ : derived reactive oxygen metabolites; BAP: biological antioxidant potential.

a single physical exercise produces reactive oxygen species (ROS) that results in oxidative stress. A previous report indicated that in exhaustive and prolonged exercise, ROS production was more elevated, which was associated with a higher degree of oxidative stress [11]. Therefore, management of physical condition, including suppression of immune function, is an essential part of fulfilling performance in athletes. Recently, salivary NO has been suggested as an index of training intensity [12]. Several studies have investigated the relationship between exercise and salivary NO levels [2, 12, 13], but only few studies have examined salivary NO levels in athletes. To use salivary NO level as a physical condition marker, the characteristics of the levels of salivary NO and oxidative stress markers in athletes must first be clarified in a large-scale cross-sectional study.

Thus, the aims of this study were, firstly to investigate the relationship between levels of salivary NO and oxidative stress markers (d-ROM and BAP); and secondly, to examine the association if any with salivary NO levels and existing diseases from past medical records in elite undergraduate athletes of public university in Japan. To achieve these aims, this research was designed as a large-scale cross-sectional study.

\section{Materials and Methods}

\section{Participants}

A total of 250 elite undergraduate athletes belonging to the faculty of health and sports science, University of Tsukuba, Ibaraki, Japan volunteered to participate in this cross-sectional study (Table 1), including 151 men and 99 women. Elite athletes were defined in this study as those who have participated in national competitions and/or have a representative experience. The details of the athletes who participated in this study have been shown in Table 2. This study was approved by the ethical committees of the Faculty of Health and Sport Sciences of the University of Tsukuba (Tai 28-25). The study was conducted in accordance with the principles outlined in the Declaration of Helsinki, and all the participants provided written informed consent before inclusion in the study.

Table 2. Salivary NO Levels of the Subjects for Each Sport Sub-Groups

\begin{tabular}{lll}
\hline Athletic events & Males & Females \\
\hline Football $(\mathrm{T}=32 ; \mathrm{M}=25, \mathrm{~F}=7)$ & $448 \pm 48$ & $337 \pm 73$ \\
Handball $(\mathrm{T}=25 ; \mathrm{M}=13, \mathrm{~F}=12)$ & $277 \pm 31$ & $433 \pm 53$ \\
Basketball $(\mathrm{T}=10 ; \mathrm{M}=0, \mathrm{~F}=10)$ & No data & $327 \pm 49$ \\
Volleyball $(\mathrm{T}=22 ; \mathrm{M}=13, \mathrm{~F}=9)$ & $384 \pm 65$ & $346 \pm 59$ \\
Athletics $(\mathrm{T}=73 ; \mathrm{M}=47, \mathrm{~F}=26)$ & $400 \pm 34$ & $379 \pm 40$ \\
Kendo $(\mathrm{T}=18 ; \mathrm{M}=8, \mathrm{~F}=10)$ & $221 \pm 22$ & $421 \pm 45$ \\
Judo $(\mathrm{T}=17 ; \mathrm{M}=12, \mathrm{~F}=5)$ & $355 \pm 38$ & $276 \pm 83$ \\
Aquatics $(\mathrm{T}=33 ; \mathrm{M}=23, \mathrm{~F}=10)$ & $448 \pm 39$ & $406 \pm 57$ \\
Tennis $(\mathrm{T}=10 ; \mathrm{M}=3, \mathrm{~F}=7)$ & $249 \pm 17$ & $377 \pm 73$ \\
Badminton $(\mathrm{T}=10 ; \mathrm{M}=7, \mathrm{~F}=3)$ & $312 \pm 73$ & $370 \pm 78$ \\
\hline
\end{tabular}

Data are shown as the mean \pm SD of the mean and expressed in $\mu \mathrm{mol} / \mathrm{L}$. $\mathrm{T}$ : the numbers of total athletes; M: the numbers of male athletes; F: the numbers of female athletes. Athletics including jump, sprint, middle, long and combined. Aquatics include swimming and water polo. 
a

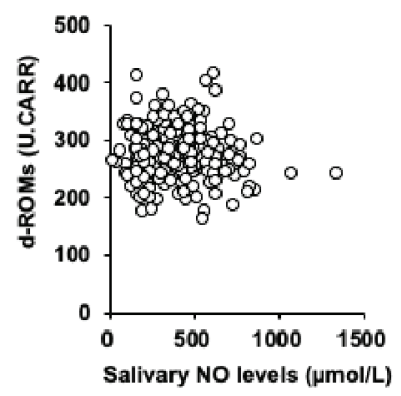

b

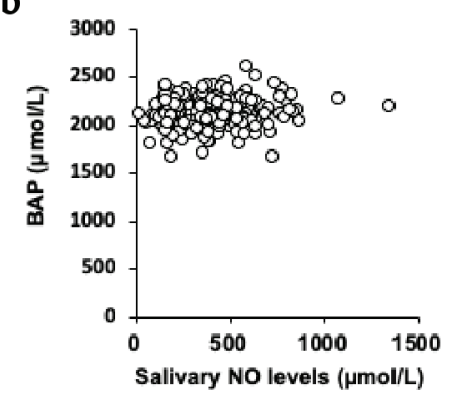

Figure 1. Univariate correlations between the levels of salivary NO and d-ROM (a) and BAP (b). NO: nitric oxide; d-ROM: derived reactive oxygen metabolites; BAP: biological antioxidant potential.

\section{Procedures}

The participants were instructed to avoid intensive exercise for at least $24 \mathrm{~h}$ before the measurements. The participants fasted overnight for $10 \mathrm{~h}$, which included suppression of caffeine consumption (allowing only caffeine-free drinks), alcohol drinking, and smoking. All measurements were conducted in a laboratory that was maintained at $24-26^{\circ} \mathrm{C}$.

\section{Questionnaire}

The participants completed the diseases questionnaire, which included the fatigue at that time and the performance over the past week, and responses were assessed using the visual analog scale (VAS). The response to each question pertaining to the athlete's condition was evaluated on the $100-\mathrm{mm}$ line of the VAS. In the questionnaire, the subjects described their past medical history and present illnesses, current medication, caffeine intake, dinner time before, and so on.

\section{Anthropometric parameters}

Anthropometric measurements were performed with the participants barefoot and wearing only light clothing. Body weight, body fat mass, skeletal muscle mass, fat-free mass, and percent body fat were measured using a body composition and body water analyzer (Inbody 770, Inbody, Seoul, Korea). To calculate the body mass index (BMI), weight $(\mathrm{kg})$ was divided by the height squared $\left(\mathrm{m}^{2}\right)$.

\section{Salivary NO levels evaluation}

Saliva samples were obtained in accordance with method previously described by Akimoto et al [14], for measurement of salivary NO levels and saliva flow rate (saliva volume). The nitrate concentration as salivary NO level was assessed using a NO detection kit (ADI-917-010, Enzo, NY, USA) with a colorimetric assay, in accordance with the manufacturer's instructions. All the samples were assayed in duplicate, and the mean absorbance value was used as the representative values.
Salivary NO levels were expressed in $\mu \mathrm{mol} / \mathrm{L}$.

\section{d-ROMs and BAP measurements}

Blood samples were obtained for measuring oxidative stress markers (d-ROM and BAP) from a fingertip into a $300-\mu \mathrm{L}$ micro tube (DI-002-06, Wismerll, Tokyo, Japan) by using a lancet (DI-003b,Wismerll, Tokyo, Japan). Plasma levels of oxidative stress markers (d-ROM and BAP) were measured with a free radical analyzer (Free Carrio Duo, Diacron International srl, Grosseto, Italy)in accordance with the method previously described [15]. The results for d-ROM were expressed in Caratelli units (U.CARR), where 1 U.CARR is equivalent to $0.08 \mathrm{mg} / \mathrm{dL}$ of $\mathrm{H}_{2} \mathrm{O}_{2}$. The results for BAP were expressed in $\mu \mathrm{mol} / \mathrm{L}$. BAP/d-ROM ratio was also measured for evaluation of serum oxidant-antioxidant balance $[16,17]$.

\section{Statistical analyses}

Statistical analyses were performed using SPSS Statistics 25.0 for Macintosh (IBM, Tokyo, Japan). The Shapiro-Wilk test was used to evaluate the normality of regression. In Table 1, the variables are expressed as mean $\pm \mathrm{SD}$. The Mann-Whitney $U$ test was used to identify sex-related differences in the participants' characteristics. In Figure 1, the Spearman's rank correlation coefficients $\left(\mathrm{r}_{\mathrm{s}}\right)$ were used to express linear correlations between the variables. In Figure 2, data are expressed as mean \pm SE. The Mann-Whitney U test was used to compare the two groups. In Figure 3, to assess the reliability of salivary $\mathrm{NO}$ levels as a prognostic variable by using receiver-operating characteristic (ROC) curves, we calculated the sensitivities and specificities in predicting asthma at cut-off values. In all the tests, a value of $\mathrm{P}<0.05$ was considered to indicate a statically significant difference.

\section{Results}

The subjects' characteristics and the sex-related differences between the two groups (men and women) in this cross-sectional study are presented in Table 1. Significant sex-related differ- 


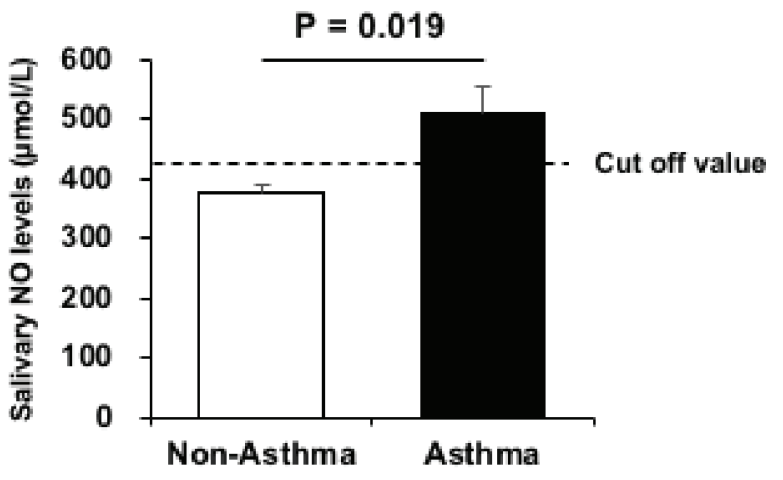

Figure 2. Salivary NO levels in the asthma (black) and non-asthma groups (White). Data are presented as mean \pm SE of the mean. NO: nitric oxide.

ences in height, weight, BMI, body fat mass, skeletal muscle mass, fat-free mass, percent body fat, d-ROM, BAP, d-ROM/ BAP ratio, fatigue scale, and performance scale were found.

Figure 4 presents a histogram of the salivary NO levels of the elite university level athletes. The salivary NO levels of athletes were the most frequent in the range of $201-300$ $\mu \mathrm{mol} / \mathrm{L}$. The number of athletes gradually decreased having salivary NO levels from the range of more than $400 \mu \mathrm{mol} / \mathrm{L}$ or more. The mean salivary NO levels of the subjects for each sport are presented in Table 2. The results of disease/illness/ health problems existing in study athletes investigation using the questionnaire have been demonstrated in Table 3. Most of the athletes in this study were healthy university students

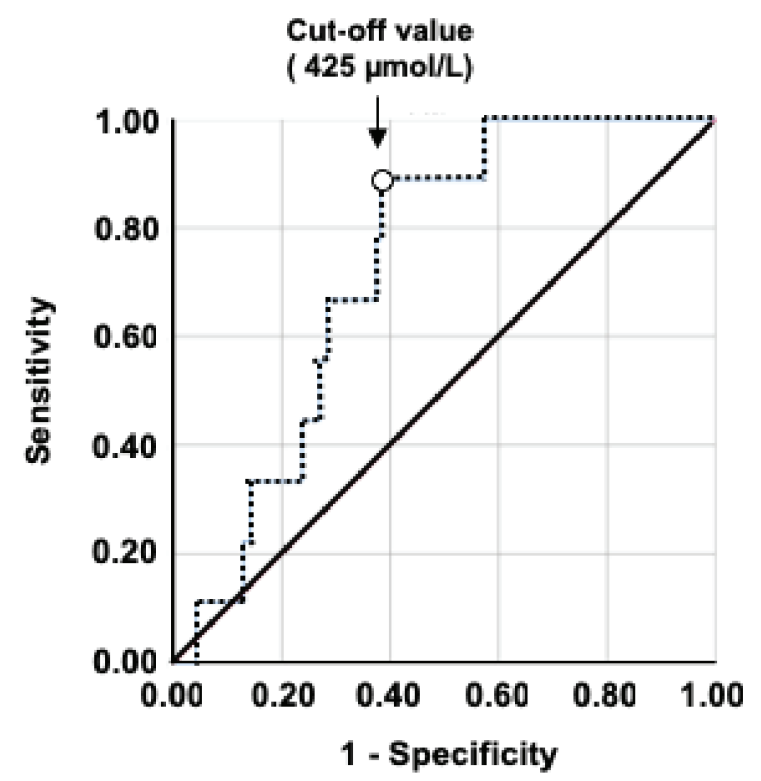

Figure 3. Receiver-operating characteristic (ROC) curve analysis of the validation data. The ROC curve of the salivary NO level is higher for asthma. The sensitivity, specificity, AUC value, and cut-off salivary NO levels are shown. NO: nitric oxide; AUC: area under the curve.

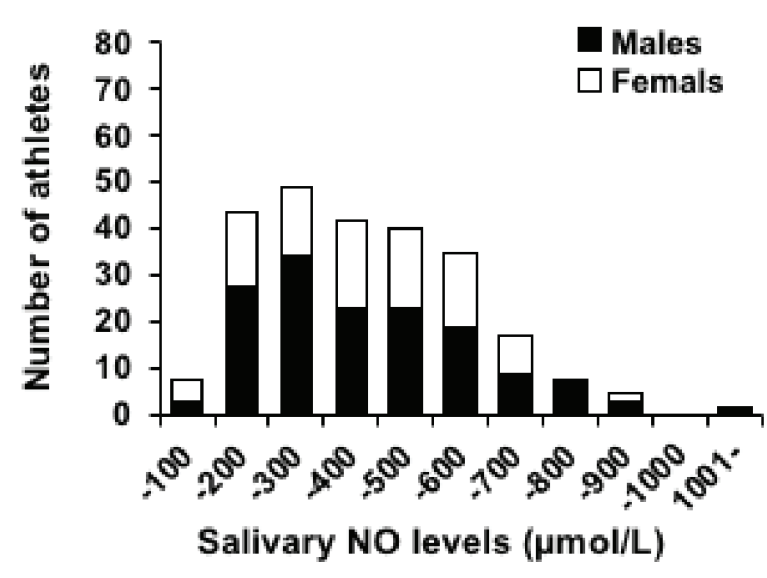

Figure 4. Histogram of the salivary NO levels of the athletes. NO: nitric oxide.

without any remarkable health problem. Twenty-six athletes (the number of total study subjects) responded that they had been diagnosed having health disorder in the past and/or present by physicians. Among them, 18 athletes (the number of study athletes having medical disorders) were found to have allergic diseases including asthma, food allergy, drug allergy, rhinitis, and dermatitis. On the other hand, eight athletes had non-allergy diseases such as anemia, arrhythmia, pneumonia, and gastroenteritis. These athletes had not another disease.

The univariate associations between the salivary NO levels and the parameters related to oxidative stress are presented in Figure 1. The salivary NO levels were not significantly associated with the levels of both d-ROM $\left(\mathrm{r}_{\mathrm{s}}=-0.054 ; \mathrm{P}=0.393\right.$; Fig. 1a) and BAP $\left(r_{s}=0.073 ; P=0.251\right.$; Fig. 1b). And, the salivary NO levels were not significantly associated with the daily practice time of both high intensity exercise $\left(r_{s}=0.065\right.$; $\mathrm{P}=0.305)$ and moderate intensity exercise $\left(\mathrm{r}_{\mathrm{s}}=-0.041 ; \mathrm{P}=\right.$ 0.532 ) and were not significantly correlated with the subjective condition markers of both fatigue $\left(\mathrm{r}_{\mathrm{s}}=0.004 ; \mathrm{P}=0.951\right)$ and performance $\left(\mathrm{r}_{\mathrm{s}}=-0.030 ; \mathrm{P}=0.637\right)$.

The difference between the two groups according to asthma history is shown in Figure 2. Salivary NO levels were significantly higher $(\mathrm{P}<0.05)$ in the group with asthma $(\mathrm{n}=$ $9 ; 510 \pm 43 \mu \mathrm{mol} / \mathrm{L})$ than in the group without asthma ( $\mathrm{n}=$ $241 ; 375 \pm 13 \mu \mathrm{mol} / \mathrm{L})$. However, d-ROM values were not significantly different $(\mathrm{P}=0.367)$ between the asthma $(285 \pm 6$ U.CARR) and non-asthma athletes groups ( $278 \pm 3$ U.CARR). Figure 3 presents the ROC curves to determine the best cut-off value of salivary NO for asthma. The optimal cut-off value $(\mathrm{P}=0.019)$ of the salivary NO for predicting asthma was 425 $\mu \mathrm{mol} / \mathrm{L}$, with a sensitivity of $88.9 \%$ and specificity of $61.8 \%$ (area under the curve (AUC), 0.73).

\section{Discussion}

This study aimed to clarify the characteristics of salivary NO levels in elite athletes of undergraduate program of Japanese 
Table 3. Detailed Information of Medical/Health Disorders Existing in Study Subjects

\begin{tabular}{lllll}
\hline \multirow{2}{*}{ Disease } & $\mathbf{n}=\mathbf{2 6}$ & \multicolumn{3}{c}{ Current symptoms } \\
\cline { 3 - 5 } & & Yes & No & Medication \\
\hline Asthma & $\mathrm{n}=9$ & $\mathrm{n}=1$ & $\mathrm{n}=8$ & $\mathrm{n}=1$ \\
Food and Drug & $\mathrm{n}=3$ & $\mathrm{n}=1$ & $\mathrm{n}=2$ & $\mathrm{n}=0$ \\
Rhinitis & $\mathrm{n}=3$ & $\mathrm{n}=3$ & $\mathrm{n}=0$ & $\mathrm{n}=2$ \\
Dermatitis & $\mathrm{n}=3$ & $\mathrm{n}=3$ & $\mathrm{n}=0$ & $\mathrm{n}=1$ \\
Non-allergy diseases & $\mathrm{n}=8$ & $\mathrm{n}=2$ & $\mathrm{n}=6$ & $\mathrm{n}=2$ \\
\hline
\end{tabular}

Non-allergy diseases: anemia, arrhythmia, pneumonia, and gastroenteritis, etc.

public university, focusing on the relationship between levels of salivary NO and oxidative stress markers (d-ROM and BAP), and various existing diseases/health disorders. In this large-scale cross-sectional study, salivary NO levels were not significantly associated with the levels of oxidative stress markers such as d-ROM and BAP. The salivary NO levels were significantly higher in the group with asthma history. From this finding, the cut-off value of salivary NO for predicting asthma was calculated to be $425 \mu \mathrm{mol} / \mathrm{L}$.

Although salivary NO levels have been reported to be related to oxidative stress $[3,4]$, no association was found with the levels of oxidative stress markers (d-ROM and BAP) in this cross-sectional study. In previous studies on the relationship between levels of d-ROM and oral inflammation [18, 19], patients with periodontitis had higher d-ROM values than control subjects [19]. The mean d-ROM level of the elite athletes in this study was lower than the reference value of 300 U.CARR, which means that oxidative stress was low. Hence, among the subjects with low oxidative stress, the relationship between the levels of oxidative stress markers and salivary NO may be difficult to confirm owing to the small individual differences in this cross-sectional study. Athletes of different ages with age-matched non-athletes should be compared in depth. If individual differences in oxidative stress are greater, the relationship between salivary NO levels and oxidative stress will be more apparent and evident. On the other hand, in this study, we only examined the cross-sectional relationship of salivary NO levels and oxidative stress. It is also necessary to examine the relationship between salivary NO levels and oxidative stress exploiting longitudinal study design.

In several studies, the response of salivary NO levels to exercise in athletes has been reported $[2,20]$, while such investigation has not been done in cross-sectional study design in athletes performing high-intensity exercise. Further, there are no studies to date reporting the distribution of salivary NO levels based on concentration on salivary NO levels in elite class Japanese university athletes. This is the first study to clarify the histogram of athletes' salivary NO levels in a large-scale cross-sectional study. This finding will contribute to establish the athlete's salivary NO standard/reference levels in Japanese athletes. Additionally, the relationship between salivary NO levels and various medical problems/diseases existing in Japanese elite athletes was unknown. Although the majority of current study subjects were healthy, but 18 athletes had allergic disorders evident from their medical history. Among them, the most confirmed allergic disorder was bronchial asthma (nine athletes). Of note, the number of study athletes having other allergic disorders was not statistically enough to be analyzed. Therefore, we focused on the details history of asthma and sub-analysis was done on the asthma group. We found that the athletes with asthma had higher salivary NO levels than the athletes without asthma. In patients with asthma, exhaled NO (FeNO) level has been used as an indicator of airway inflammatory process [21]. FeNO was reported to be derived from iNOS as well as salivary NO [22]. Furthermore, iNOS expression increases inflammatory salivary gland secretions [23]. Thus, salivary NO level also has the possibility of reflecting airway inflammation, including asthma, and FeNO. Whereas FeNO has clear reference values for airway inflammation [21], while salivary NO has no reference values for diseases. Hence, in this study, the cut-off value of salivary NO for predicting asthma was calculated using the ROC curve. As a result, we found that $425 \mu \mathrm{mol} / \mathrm{L}$ is the cut-off value for predicting asthma in this study. In the histogram of salivary NO levels, the frequency steadily decreases from $>400 \mu \mathrm{mol} / \mathrm{L}$. The sensitivity $(88.9 \%)$ at the cut-off value was higher than the specificity $(61.8 \%)$, which means that although the risk of failure to notice asthma is low, the high possibility of considering a nonasthmatic patient as an asthmatic patient must be kept in mind. On the other hand, the levels of d-ROM in asthma were associated with the degree of airway obstruction [24]. However, the levels of d-ROM in this study were not associated with asthma. Salivary NO levels could possibly identify asthmatic patients overlooked when d-ROM value was used. Therefore, at present, combining levels of salivary NO and FeNO as an index of asthma compensates for the low specificity.

In addition, the significant sex-related differences were observed in the various measurements in this study (Table 1). Both oxidative stress markers (d-ROM, BAP, and d-ROM/ $\mathrm{BAP}$ ratio) and subjective indicators (fatigue and performance) significantly differed by sex. The male athletes had better d$\mathrm{ROM} / \mathrm{BAP}$ ratio, less subjective fatigue, and higher subjective performance than the female athletes. The sex-related difference in oxidative stress should consider the effect of menstruation, a physiological phenomenon peculiar to women. In other words, sex hormones may have affected this sex-related difference. Estrogen, as sex hormones, has been reported to have an antioxidant activity [25]. During the menstrual cycle, the estrogen level is low value in the menstrual phase [26]. Athletic performance is influenced by symptoms occurring before 
menstruation $[27,28]$. Thus, the reason for the higher levels of oxidative stress markers (d-ROM, BAP, and d-ROM/BAP ratio) and subjective markers (fatigue and performance) in the female athletes than in the male athletes might be because most of the female athletes were in the menstrual phase. However, the levels of sex hormones such as estrogen could not be measured in this study to determine the menstrual phase. In further research, the menstrual cycle in female athletes must be identified and the differences in menstrual phase in female athletes and oxidative stress markers between the sexes must be examined.

\section{Limitations}

One of the potential study limitations to be mentioned is the absence of non-athlete study group in the current research design which prevented us from performing many sub-analyses on the characteristics of salivary NO levels in elite class university athletes in Japan. Additionally, the present study has other several noteworthy limitations as follows: 1) The age range was limited to the undergraduate university program level; 2) A longitudinal study was not conducted on the relationship between asthma and salivary NO levels; 3) The mechanisms underlying the response of salivary NO level to respiratory inflammation were not investigated; 4) The oxidative stress markers used to examine the relationship with salivary NO levels in this study were only d-ROM and BAP; 5) We could not measure the levels of sex hormones such as estrogen for gender based interpretation; and 6) Finally training information was collected and evaluated only through questionnaire method. A longitudinal study with a larger sample size, including other age groups, to establish the usefulness of salivary NO level as a disease marker and experimental basic research to identify the mechanism of salivary NO for asthma is highly required and warranted in future.

\section{Conclusions}

In this large-scale cross-sectional study, the salivary NO levels were not associated with the levels of d-ROM and BAP, which are oxidative stress markers in Japanese university undergraduate level elite athletes. On the other hand, bronchial asthma was the most prevalent medical disorder in this studied population and the salivary NO levels were higher in the group with asthma than in comparison to non-asthma group. The current findings indicate that salivary NO levels may be a marker that potentially reflects/predicts asthma in Japanese young elite athletes in a hope to establish salivary NO level as a respiratory tract condition marker in athletes.

\section{Acknowledgments}

We are grateful to Hiroshi Kumagai, Koichiro Tanahashi, Keita Suzuki, and Momoko Endo, for their technical assistance and helpful discussions. And, we would like to thank Editage (www.editage.jp) for English language editing.

\section{Conflict of Interest}

No potential conflict of interest was disclosed.

\section{Grant Support}

This research received a Grant-in-Aid for the Japan Society for the Promotion of Science Fellows (KAKENHI, grant No. 18K10810).

\section{References}

1. Nathan C. Nitric oxide as a secretory product of mammalian cells. FASEB J. 1992;6(12):3051-3064.

2. Panossian AG, Oganessian AS, Ambartsumian M, Gabrielian ES, Wagner H, Wikman G. Effects of heavy physical exercise and adaptogens on nitric oxide content in human saliva. Phytomedicine. 1999;6(1):17-26.

3. Takahama U, Hirota S, Nishioka T, Oniki T. Human salivary peroxidase-catalyzed oxidation of nitrite and nitration of salivary components 4-hydroxyphenylacetic acid and proteins. Arch Oral Biol. 2003;48(10):679-690.

4. Takahama U, Hirota S, Oniki T. Quercetin-dependent scavenging of reactive nitrogen species derived from nitric oxide and nitrite in the human oral cavity: interaction of quercetin with salivary redox components. Arch Oral Biol. 2006;51(8):629-639.

5. Dervisevic A, Babic N, Huskic J, Sokolovic S, NakasIcindic E, Causevic L. Concentration of nitric oxide in saliva of patients with rheumatoid arthritis. Int J Collab Res Intern Med Public Health. 2012;4(7):1442-1451.

6. Reher VG, Zenobio EG, Costa FO, Reher P, Soares RV. Nitric oxide levels in saliva increase with severity of chronic periodontitis. J Oral Sci. 2007;49(4):271-276.

7. Cacciapaglia F, Grazia Anelli M, Rizzo D, Morelli E, Mazzotta D, Scioscia C, Iannone F, et al. Effective tumour necrosis factor-blocking therapy reduces reactive oxygen metabolite level in rheumatoid arthritis. J Int Med Res. 2016;44(1 suppl):28-32.

8. Morimoto M, Satomura S, Hashimoto T, Ito E, Kyotani S. Oxidative stress measurement and prediction of epileptic seizure in children and adults with severe motor and intellectual disabilities. J Clin Med Res. 2016;8(6):437-444.

9. Klentrou P, Cieslak T, MacNeil M, Vintinner A, Plyley M. Effect of moderate exercise on salivary immunoglobulin A and infection risk in humans. Eur J Appl Physiol. 2002;87(2):153-158.

10. Nieman DC. Exercise, infection, and immunity. Int J Sports Med. 1994;15(Suppl 3):S131-141.

11. Ilhan N, Kamanli A, Ozmerdivenli R, Ilhan N. Variable effects of exercise intensity on reduced glutathione, thiobarbituric acid reactive substance levels, and glucose concentration. Arch Med Res. 2004;35(4):294-300. 
12. Diaz MM, Bocanegra OL, Teixeira RR, Soares SS, Espindola FS. Salivary nitric oxide and alpha-amylase as indexes of training intensity and load. Int J Sports Med. 2013;34(1):8-13.

13. Rahman ZA, Abdullah N, Singh R, Sosroseno W. Effect of acute exercise on the levels of salivary cortisol, tumor necrosis factor-alpha and nitric oxide. J Oral Sci. 2010;52(1):133-136.

14. Akimoto T, Kumai Y, Akama T, Hayashi E, Murakami H, Soma R, Kuno S, et al. Effects of 12 months of exercise training on salivary secretory IgA levels in elderly subjects. Br J Sports Med. 2003;37(1):76-79.

15. Morimoto M, Hashimoto T, Kitaoka T, Kyotani S. Impact of oxidative stress and newer antiepileptic drugs on the albumin and cortisol value in severe motor and intellectual disabilities with epilepsy. J Clin Med Res. 2018;10(2):137-145.

16. Iimura Y, Agata U, Takeda S, Kobayashi Y, Yoshida S, Ezawa I, Omi N. Lycopene intake facilitates the increase of bone mineral density in growing female rats. J Nutr Sci Vitaminol (Tokyo). 2014;60(2):101-107.

17. Fukui T, Maruyama M, Yamauchi K, Yoshitaka S, Yasuda T, Abe Y. Effects of febuxostat on oxidative stress. Clin Ther. 2015;37(7):1396-1401.

18. Tamaki N, Tomofuji T, Ekuni D, Yamanaka R, Morita M. Periodontal treatment decreases plasma oxidized LDL level and oxidative stress. Clin Oral Investig. 2011;15(6):953-958.

19. Tamaki N, Tomofuji T, Ekuni D, Yamanaka R, Yamamoto T, Morita M. Short-term effects of non-surgical periodontal treatment on plasma level of reactive oxygen metabolites in patients with chronic periodontitis. J Periodontol. 2009;80(6):901-906.
20. Ulkar B, Elgun S, Ozmeric N, Ozdemir B, Boynuegri D. Periodontal nitric oxide pathway alteration due to precompetition anxiety in handball players. J Periodontol. 2012;83(2):204-210.

21. Dweik RA, Boggs PB, Erzurum SC, Irvin CG, Leigh MW, Lundberg JO, Olin AC, et al. An official ATS clinical practice guideline: interpretation of exhaled nitric oxide levels (FENO) for clinical applications. Am J Respir Crit Care Med. 2011;184(5):602-615.

22. Ricciardolo FL, Sterk PJ, Gaston B, Folkerts G. Nitric oxide in health and disease of the respiratory system. Physiol Rev. 2004;84(3):731-765.

23. Correia PN, Carpenter GH, Paterson KL, Proctor GB. Inducible nitric oxide synthase increases secretion from inflamed salivary glands. Rheumatology (Oxford). 2010;49(1):48-56.

24. Nakamoto K, Watanabe M, Sada M, Inui T, Nakamura M, Honda K, Wada H, et al. Serum Reactive Oxygen Metabolite Levels Predict Severe Exacerbations of Asthma. PLoS One. 2016;11(10):e0164948.

25. Kendall B, Eston R. Exercise-induced muscle damage and the potential protective role of estrogen. Sports Med. 2002;32(2):103-123.

26. Sawai A, Tochigi Y, Kavaliova N, Zaboronok A, Warashina Y, Mathis BJ, Mesaki N, et al. MRI reveals menstruallyrelated muscle edema that negatively affects athletic agility in young women. PLoS One. 2018;13(1):e0191022.

27. Lebrun CM. Effect of the different phases of the menstrual cycle and oral contraceptives on athletic performance. Sports Med. 1993;16(6):400-430.

28. Bale P, Nelson G. The effects of the menstruation on performance of swimmers. Aust J Sci Med Sport. 1985;17(1):19-22. 